\title{
DRENAJE GRAVITACIONAL ASISTIDO CON VAPOR, SAGD, APLICADO A YACIMIENTOS DE CRUDOS PESADOS
}

Astrid Xiomara Rodriguez Castelblanco ${ }^{1 *}$, Jorge Mario Palma Bustamante ${ }^{2}$, Samuel Fernando Muñoz Navarro ${ }^{3}$

*A quien debe dirigirse la correspondencia

\section{RESUMEN}

A nivel mundial, se están buscando nuevas técnicas de recobro mejorado para implementarlas en yacimientos de crudo pesado, ya que la mayoría de las reservas a nivel mundial son de este tipo de hidrocarburo. Un método de recobro actualmente existente es el de drenaje gravitacional asistido con vapor, SAGD, el cual ha sido implementado en yacimientos con bitumen, donde se han alcanzado resultados exitosos, obteniendo factores de recobro superiores al 50\%.

La técnica consiste en dos pozos horizontales uno ubicado a pocos pies sobre el otro dependiendo de la viscosidad del hidrocarburo, en el cual, por el pozo superior se hace la inyección continua de vapor donde por diferencias de densidades el vapor tiende a expandirse hasta el tope de la formación y los hidrocarburos calentados y el vapor de agua condensado drenan hacia el pozo inferior, que es el productor. El mecanismo de producción que se evidencia en esta técnica es la segregación gravitacional, ya que como es aplicada a yacimientos con bitúmenes sólo fluirán los hidrocarburos calentados hacia el pozo productor por efecto de la gravedad, por lo cual es necesario que exista una conectividad entre los dos pozos.

Sin embargo, en la mayoría de yacimientos de crudo pesado el hidrocarburo presenta movilidad, es decir, la viscosidad no es tan alta $(<10.000 \mathrm{cP})$, para los cuales la técnica SAGD no es exitosa, a causa de que en estos yacimientos no solo influyen las fuerzas gravitacionales sino que también las fuerzas viscosas, por lo cual, el vapor inyectado se expande hacia el overburden, canalizando el vapor, presentando pérdidas de energía y formación de emulsiones, lo cual hace que la cámara de vapor, la cual es el principio físico de la técnica SAGD no se genere.

En este artículo se presenta una comparación del comportamiento y desarrollo de la cámara de vapor en dos yacimientos con las mismas características, diferenciados por el tipo de fluidos, pues uno de ellos está saturado con crudo extra pesado $(254,814 \mathrm{cP})$ y el otro saturado con crudo pesado móvil (4019.6 cP), bajo el cual se evidencia el overriding del vapor en el crudo móvil a causa de la movilidad del crudo y de las fuerzas viscosas.

Palabras clave: Crudos Extra Pesados, Cámara de Vapor, Drenaje gravitacional.

1. MSc. En Ingeniería de Hidrocarburos. Escuela de Ingeniería de Petróleos. Universidad Industrial de Santander. Bucaramanga.Colombia. asxiorod@uis.edu.co

2. MSc. En Ingeniería de Hidrocarburos. Ecopetrol.S.A. Colombia. Jorge.palma@ecopetrol.com.co

3. MSc. En Ingeniería de Hidrocarburos. Escuela de Ingeniería de Petróleos. Universidad Industrial de Santander. Bucaramanga.Colombia.samuel@uis.edu.co 


\section{STEAM ASSISTED GRAVITY DRAINAGE, SAGD, APPLIED TO HEAVY OIL RESERVOIRS}

\section{ABSTRACT}

Worldwide, new techniques to implement Enhanced Oil Recovery in heavy oil reservoirs have been studying, since as most of the global reserves are of this type of hydrocarbon. The Steam Assisted Gravity Drainage (SAGD) is a cutting age EOR method that have been applied in bitumen reservoirs gaining successful results, obtaining recovery factors up to $50 \%$.

The technique involves two horizontal wells, the first one located a few feet above the second one depending on the viscosity of the crude oil; by the upper well, the continuous vapor injection is made an for density differences this vapor tents to expand to the top of the formation heating the hydrocarbons which then, by gravity come down in the reservoir to be produce, with the condensed vapor, through the second horizontal well. The production mechanism evidenced in this technique is the gravity drainage because in its implementation, applied to bitumen, only the heated hydrocarbons will flow through the producer well by the effect of the gravity therefore a connectivity between the two wells must be necessary.

However, in the majority of heavy oil reservoirs the crude have mobility i.e. the viscosity is not to high $(<10.000$ $\mathrm{cP}$ ), at which the SAGD technique is not effective. This is because in this reservoirs no only the gravitational forces have influence, the viscous forces does to, whereby the injected vapor expands to the overburden, canalizing the vapor, generating energy losses and emulsions formation, causing that the vapor chamber, which is the physical principle of the SAGD, not to form.

This paper presents a comparison of the behavior and develop of the vapor chamber in two different reservoirs with the same characteristics but different type of fluids, the first one is saturated with an extra heavy oil (254814 cP) and the other saturated with a heavy oil with mobility $(4019.6 \mathrm{cP})$ in which the overriding of the vapor in the mobile oil by the effect of the gravitational forces is observed.

Keywords: Heavy Oil, SAGD, Gravity Drainage, Steam Injection, Steam Chamber.

\section{INTRODUCCIÓN}

La técnica de drenaje gravitacional asistida con vapor fue desarrollada para aplicarla a crudos extrapesados con viscosidades superiores a $10.000 \mathrm{cP}$, en los cuales se ha logrado recuperar más del $50 \%$ de las reservas existentes, sin embargo a nivel mundial se encuentran numerosos yacimientos de crudos pesado con movilidad donde su factor de recobro es muy bajo, por lo cual es necesario evaluar el desempeño de esta técnica en estos tipos de yacimiento para poder determinar las mejores condiciones de implementación.

La herramienta utilizada para el desarrollo y evaluación del proceso SAGD en los dos tipos de crudos fue STARS de la compañía CMG, la cual nos permite representar las pérdidas que se generan en la tubería de inyección y el fenómeno de la cámara de vapor y de la piscina de líquidos.

A partir de este estudio se puede evidenciar la necesidad de ajustar la técnica para poder ampliar el rango de aplicabilidad del proceso y así poder aplicarla en mayor número de campos y obtener mayor cantidad de reservas en superficie a condiciones económicas favorables.

\section{DRENAJE GRAVITACIONAL ASISTIDO CON VAPOR}

La técnica de drenaje gravitacional asistida con vapor SAGD, se basa principalmente en la creación y expansión de la cámara de vapor bajo un único mecanismo de producción, la Drenaje Gravitacional. En este proceso, el vapor el cual es continuamente inyectado a la formación mediante un pozo horizontal se expande uniformemente, calentando el hidrocarburo; de esta manera el crudo adquiere movilidad y drena hacia el pozo productor, ubicado a pocos pies por debajo del pozo inyector, por gravedad. Los procesos influyentes en el proceso se pueden observar en la figura 1, donde se evidencia una interface entre el vapor inyectado y el yacimiento, la cual es llamada zona de transición bajo la cual drenan los fluidos calentados y el vapor condensado. 


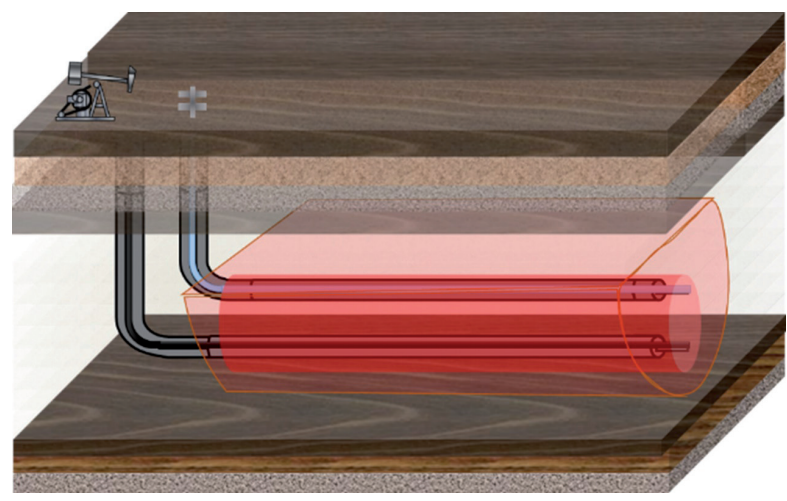

Figura 1. Diagrama del proceso de segregación gravitacional asistido con vapor.

Para el desarrollo del proceso es necesaria la creación de una piscina de líquidos, la cual consiste en un volumen de fluido líquido existente entre los dos pozos para evitar el bypass del vapor inyectado. Después de que la cámara alcanza el tope de la formación, esta continúa expandiéndose hasta alcanzar su límite económico, el cual se estima que ocurre cuando se ha alcanzado una relación vapor-aceite superior a cuatro $(\mathrm{SOR}>4)$, por tal razón Butler mediante la Teoría de Tandrain aconseja que el espaciamiento que debe existir entre pares de pozos debe ser $3 / 2$ el espesor de la formación.

La técnica se desarrolla principalmente en tres etapas, la primera de Start up, seguida por la fase de desarrollo y culminando con la etapa de mejoramiento. En la etapa inicial, el vapor es inyectado por el tubing de los dos pozos, tanto el productor como el inyector, y al mismo tiempo se presenta una producción pequeña de aceite por el anular de los mismos. Esta etapa se hace con el propósito de lograr una conectividad entre los pozos y que el crudo calentado pueda desplazarse hacia el pozo productor. Cuando se aplica SAGD a un crudo pesado móvil esta etapa no es necesaria, ya que el crudo ya tiene la facilidad de drenar hacia el pozo productor.

Después de haber alcanzado la conectividad entre pozos se continua con la etapa de desarrollo, la cual se sabe que inicia, cuando la producción de aceite en el pozo inyector disminuye, a partir de esto se continua con la inyección continua de vapor por el pozo superior y los fluidos calentados y el agua condensada drenan hacia el pozo productor por gravedad. A inicio de esta fase la cámara de vapor asciende rápidamente hasta alcanzar el tope de la formación, generando un aumento gradual de la tasa de producción de aceite, posteriormente la expansión se da lateralmente, proceso en el cual se alcanzan las tasas de producción de aceite más altas, por lo cual, en esta fase se determina la rentabilidad del proyecto, finalmente, llega un punto de la expansión en la cual las tasas de producción de aceite empiezan a declinar alcanzando el limite económico. Por tal razón, existe la tercera etapa llamada de mejoramiento, la cual propone generar gradientes de presión entre pares de pozos para que una cámara de vapor se expanda más rápido que la otra y de esta manera poder barrer mayor área del yacimiento.

\section{MODELOS DE SIMULACION DE YACIMIENTOS CON CRUDO EXTRAPESADO Y PESADO MOVIL}

Con el propósito de comparar el comportamiento del proceso SAGD en los dos crudos, se crearon dos modelos de simulación con las mismas propiedades petrofísicas, diferenciándose el uno del otro por el modelo de fluidos, pues se representa un crudo con una viscosidad de $254,814 \mathrm{cP}$ y el otro con $4019.6 \mathrm{cP}$. Las dimensiones del grid se pueden observar en la figura 2, la cual tiene $2200 \mathrm{ft}$ en la dirección i, $200 \mathrm{ft}$ en la dirección j y $80 \mathrm{ft}$ de espesor.

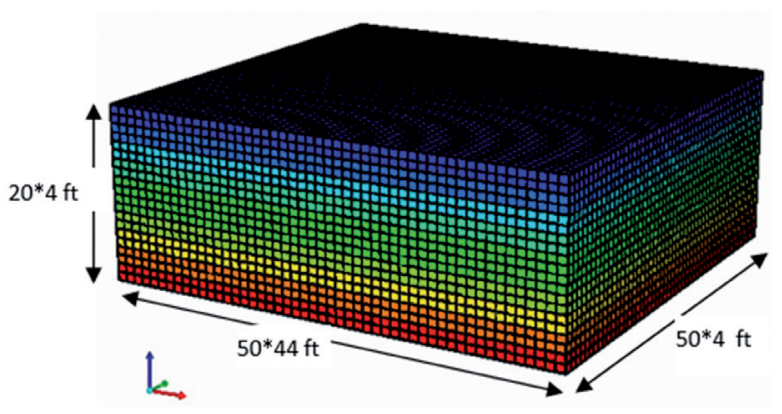

Figura 2. Grid de simulación

Fuente: Builder, CMG

La relación existente entre los espesores de las celdas de la dirección $\mathrm{k}$ y la sección transversal al pozo deben ser de uno, para poder observar en detalle la expansión de la cámara de vapor. En cuanto a las propiedades petrofísicas de la formación, se asignó una permeabilidad horizontal de $5 \mathrm{D}$ con un $\mathrm{Kv} / \mathrm{KH}$ de 0.5 para que el yacimiento permita el crecimiento vertical y la expansión lateral de la cámara de vapor y a la vez el drenaje gravitacional de los fluidos. La porosidad de la formación es del $30 \%$ lo cual conlleva a que el modelo tenga una alta saturación de aceite posible de recuperar.

MODELO DE FLUIDOS: Como son dos crudos diferentes que se van a comparar, donde un hidrocarburo es móvil y el otro no, fue necesario la creación de dos modelos de fluidos, con características diferentes, 
$\mathrm{y}$ por ende, diferentes temperaturas, presiones $\mathrm{y}$ profundidades. En la tabla 1 se presentan los datos del modelo de fluidos para los dos modelos creados.

Tabla 1: Modelos de fluidos para el crudo extra pesado y el pesado con movilidad

\begin{tabular}{|ccc|}
\hline PROPIEDAD & $\begin{array}{r}\text { CRUDO } \\
\text { EXTRA } \\
\text { PESADO }\end{array}$ & $\begin{array}{c}\text { CRUDO } \\
\text { PESADO }\end{array}$ \\
\hline Temperatura $\left({ }^{\circ} \mathrm{F}\right)$ & 53.6 & 105 \\
\hline Presión de formación (psi) & 290.1 & 900 \\
\hline Profundidad (ft) & 670 & 2000 \\
\hline${ }^{\circ}$ API & 6 & 13 \\
\hline Presión de burbuja (psi) & 116 & 540 \\
\hline $\begin{array}{c}\text { Viscosidad a Temperatura de } \\
\text { yacimiento }\end{array}$ & 254814 & 4019.6 \\
\hline
\end{tabular}

Las curvas de permeabilidad relativa utilizadas en los dos modelos se representan en la figura 3 , donde se define una saturación de agua irreducible de 0.2 y una saturación de aceite residual de 0.2 .

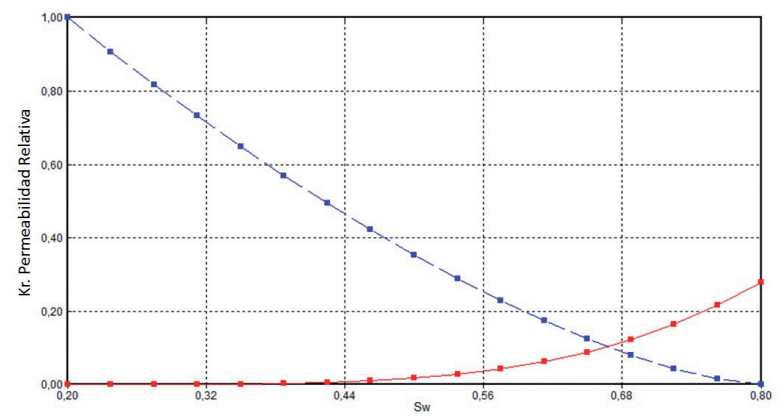

Figura 3. Curva de permeabilidad relativa

Fuente: Builder, CMG

POZOS: En los dos modelos de simulación se perforaron dos pozos horizontales de $1848 \mathrm{ft}$ de longitud. Como el yacimiento no tiene acuífero el pozo productor es perforado a $12 \mathrm{ft}$ del fondo de la formación y el inyector para el crudo extra pesado está a $16 \mathrm{ft}$ del productor y para el crudo pesado con movilidad está a $40 \mathrm{ft}$ del productor. Esta diferencia entre espaciamientos está en función de la viscosidad del hidrocarburo. Cabe aclarar que para el modelo del crudo pesado con movilidad no fue necesaria la etapa de inicialización ya que en este modelo ya existe conectividad entre pozos debido a la movilidad del crudo. A los dos modelos se les inyectó la misma cantidad de vapor, pero a diferentes presiones y temperaturas, pues se debe considerar la profundidad a la cual se encontraban los pozos. En la figura 4 se presenta un esquema de la ubicación de los pozos para el crudo extra pesado y en la figura 5 para el crudo pesado móvil.

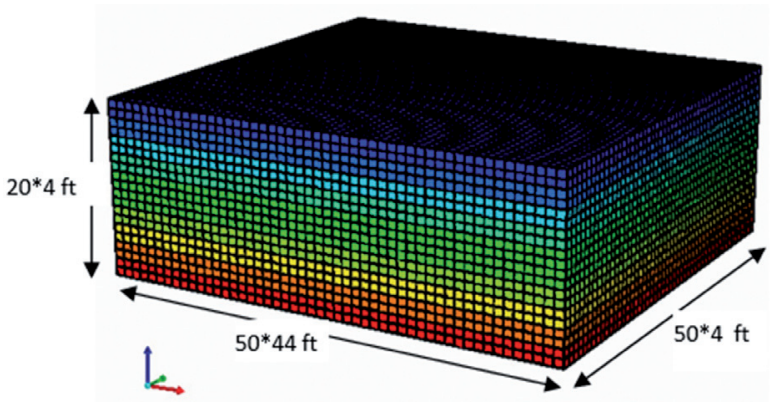

Figura 4. Configuración de pozos del modelo de crudo extrapesado

Fuente: Builder, CMG

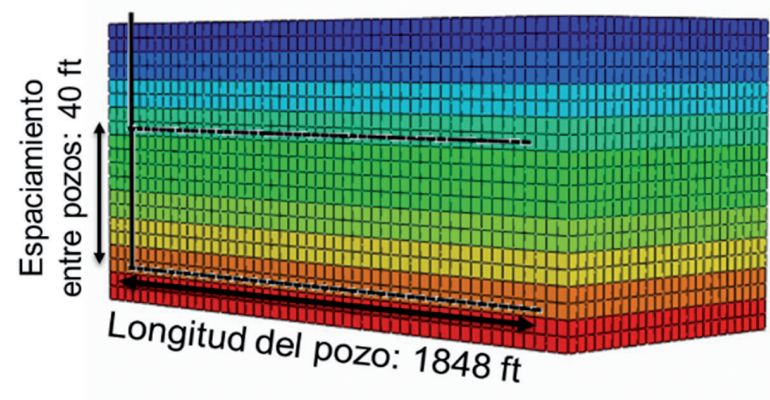

Figura 5. Configuración de pozos del modelo de crudo pesado móvil

Fuente: Builder, CMG

\section{RESULTADOS}

Los dos modelos se desarrollan y evalúan desde marzo del 2014, pero su periodo termina hasta que el vapor alcance los límites del piloto, por lo cual para cada modelo el tiempo de análisis es diferente, pues al no haber mayor longitud para la expansión el vapor, este comenzará a bajar disminuyendo la saturación del aceite remanente en las zonas inicialmente no contactadas y que en teoría nunca contactaría si se evaluara a nivel de sector model.

MODELO DEL CRUDO EXTRAPESADO: A continuación, en la figura 6 se presentan imágenes del proceso SAGD en el crudo extrapesado, por lo cual se evaluará una sección lateral y varias transversales a los pozos. Este modelo será evaluado hasta el primero de marzo de 2020, fecha en la cual se alcanzan los límites del modelo.

Las cuatro imágenes presentadas en la figura 6 corresponden al perfil de temperatura del yacimiento observado desde secciones longitudinales y transversales a los pozos. En la figura anterior se presenta una vista longitudinal de los pozos (6-a) donde se evidencia la 
uniforme inyectividad a lo largo del pozo. En la figura 6-b se puede observar que después de tres meses del proceso, en los cuales se inyecta vapor y se produce hidrocarburo, vapor y condensados en los dos pozos, se alcanza la conectividad entre estos y por lo tanto el fluido empezará a drenar hacia al pozo inferior que es el productor, finalizando el periodo de inicialización. Posteriormente la cámara comienza a expandirse uniformemente hasta alcanzar el tope de la formación (6-c), lo cual se evidencia al año de haber iniciado el proyecto donde la expansión lateral es mínima, pues inicialmente el vapor se expande verticalmente. Posterior a esto, el crecimiento de la cámara se da lateralmente hasta alcanzar el límite del piloto en estudio, que en este caso se refiere a $200 \mathrm{ft}$ de longitud, por lo cual el proceso solo se evaluará durante 6 años, porque posterior a este, el vapor ya no puede seguir expandiéndose lateralmente entonces empezará a llenar el yacimiento, efecto que realmente no sucederá en campo, pues el vapor siempre tendrán área de desplazamiento y el proyecto se vería interrumpido únicamente por alcanzar su límite económico.

Al analizar el comportamiento de la presión del yacimiento, Figura 7, se puede observar que inicialmente se ve un aumento de esta debido a las altas tasas de inyección, pero posterior a esto, la presión tiende a permanecer constante a razón de que el volumen inyectado es prácticamente igual al producido, evidenciándose que el único mecanismo influyente en la producción es la segregación gravitacional.

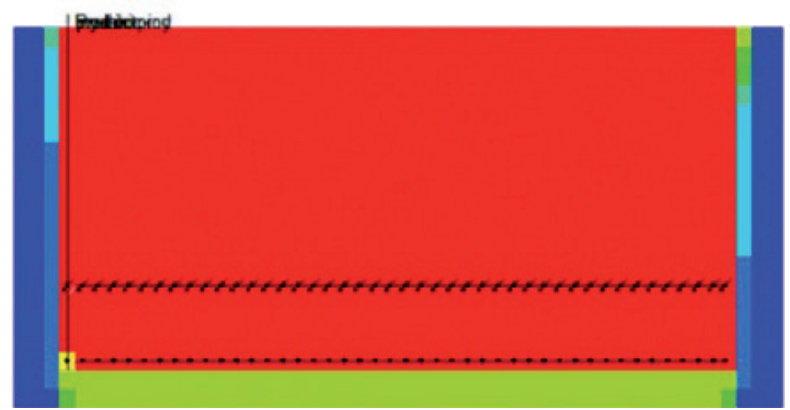

a) Seccion longitudinal de los pozos

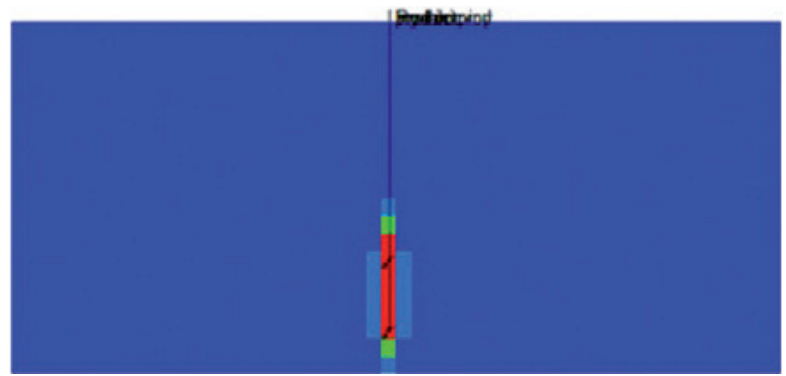

b) A los 3 meses de iniciado el proyecto.

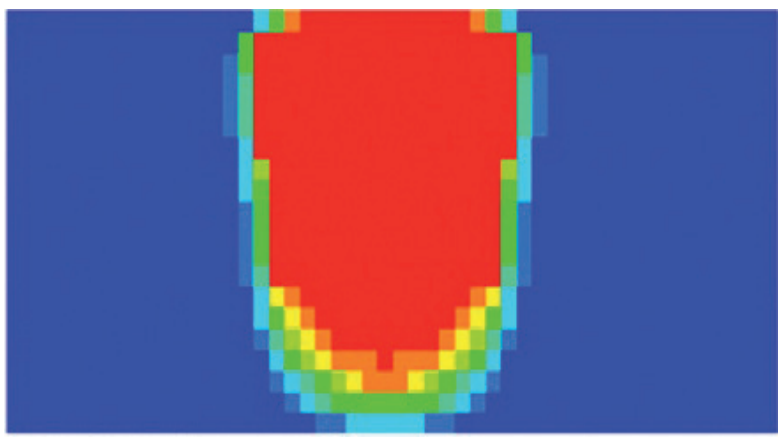

c) Al año del proceso

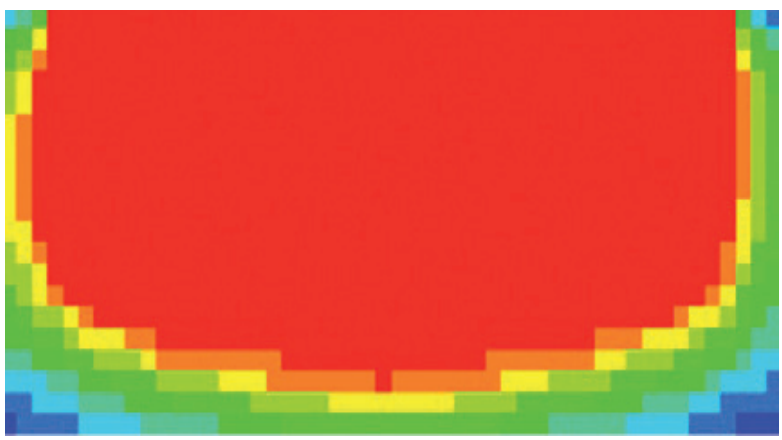

d) A los 6 años del proceso

Figura 6. Proceso SAGD implementado en el crudo extrapesado

Fuente: Results 3D, CMG

De igual manera, al analizar el factor de recobro a los seis años de implementado el proceso, figura 8 , vemos que este logra un $66 \%$, lo cual evidencia que el proceso es bastante eficiente técnicamente y al relacionarlo con el SOR (Relación vapor - aceite) el cual se mantiene en cuatro, podemos inducir que económicamente también lo es atractivo.

Presion Vs Tiempo

Modelo de crudo Extrapesado

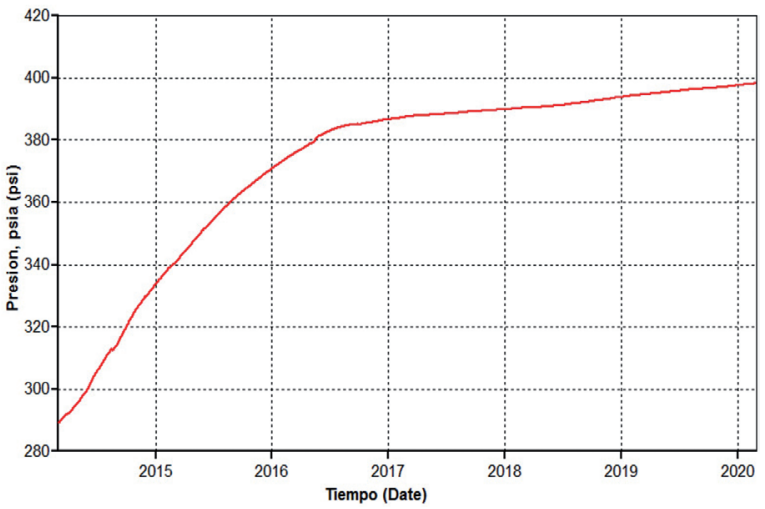

Figura 7. Comportamiento de la presión Vs tiempo, para el yacimiento de crudo extrapesado

Fuente: Results Graph, CMG 


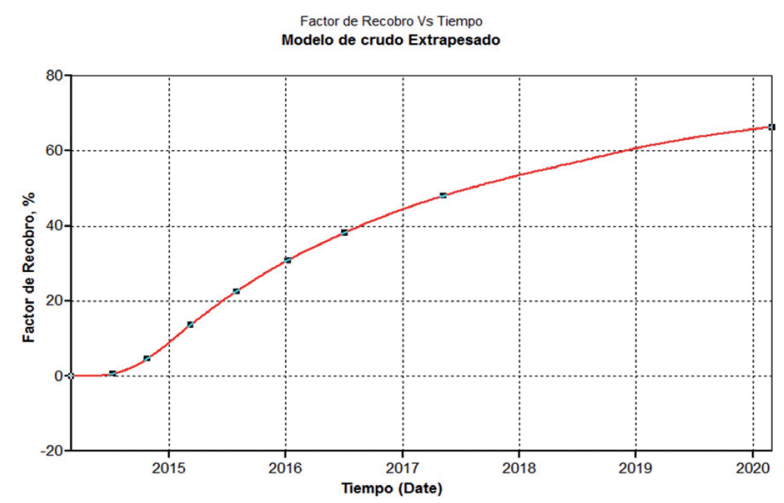

Figura 8. Factor de recobro Vs tiempo para el yacimiento de crudo extrapesado

Fuente: Results Graph, CMG

\section{MODELO DEL CRUDO PESADO CON}

MOVILIDAD: Como se mencionó anteriormente, los dos modelos tienen las mismas propiedades petrofísicas, lo que hace diferente a un modelo respecto al otro es su modelo de fluidos, la profundidad a la que se encuentra el yacimiento y el espaciamiento entre pozos. A continuación, en la figura 9, se presenta un perfil térmico de la expansión del vapor de la formación.

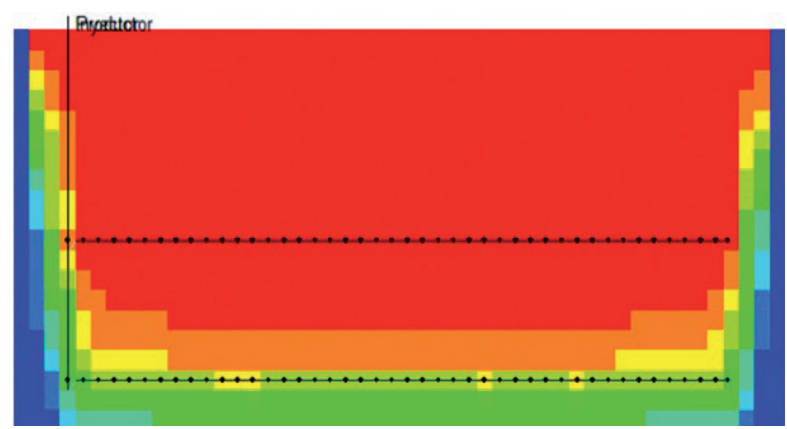

a) Seccion longitudinal de los pozos

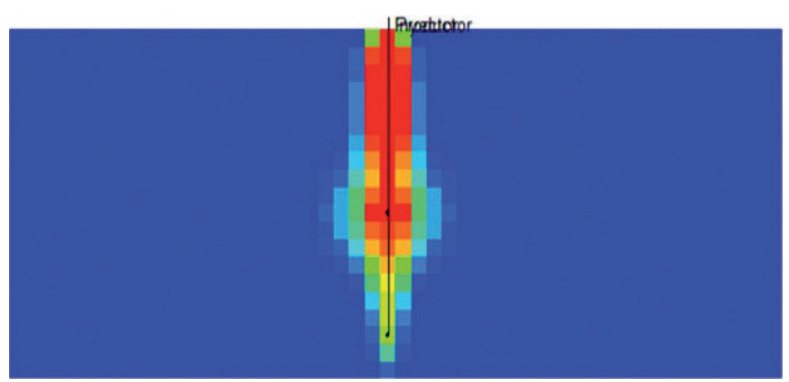

b) A los 4 meses de iniciado el proyecto.

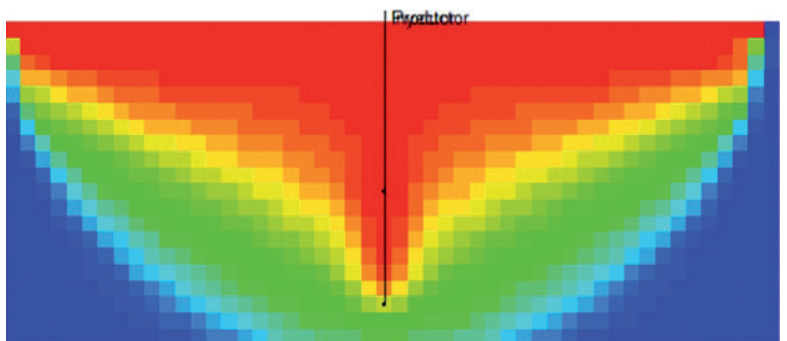

c) A los 5 años del proceso

Figura 9. Proceso SAGD implementado en un yacimiento con crudo pesado móvil

Fuente: Results Graph, CMG

Como se puede observar, para el crudo pesado con movilidad no era necesario realizarle el periodo de Start Up, pues al no ser tan viscoso el hidrocarburo, este podría desplazarse hacia el pozo productor desde el inicio. Sin embargo, el proyecto inicia a la misma fecha del extrapesado, es decir en Marzo del 2014 para tener un referente de comparación. Si analizamos detalladamente la figura 9-b, observamos que a diferencia del crudo extrapesado, en este modelo el vapor se expande tanto vertical como lateralmente, pero esta expansión no es uniforme, es más notoria en las cercanías del pozo inyector. Al analizar el comportamiento del vapor en la figura 9-c, se observa que la cámara de vapor no se forma y el vapor se expande rápidamente por el tope de la formación, dando evidencia del overriding del mismo, por lo cual el modelo es evaluado solamente hasta el año 2019, año en el cual alcanza los límites del piloto.

En la figura 10 también se observa que la presión en la formación tiende a permanecer constante, sin embargo, se debe tener en cuenta que en este modelo las fuerzas viscosas también generan influencia sobre la producción del hidrocarburo, además de las fuerzas gravitacionales.

Ya al analizar el factor de recobro del proceso, figura 11, se evidencia que este solo alcanza un $41 \%$ durante los 5 años de desarrollo, eso debido a que el área contactada y alterada por el vapor inyectado es mínima, pues el vapor se expande rápidamente hacia el overburden y no genera un eficiente barrido volumétrico. Aunque ese factor de recobro es un valor alto, este podría ser mayor si se logrará formar la cámara de vapor característica de un proceso SAGD. 


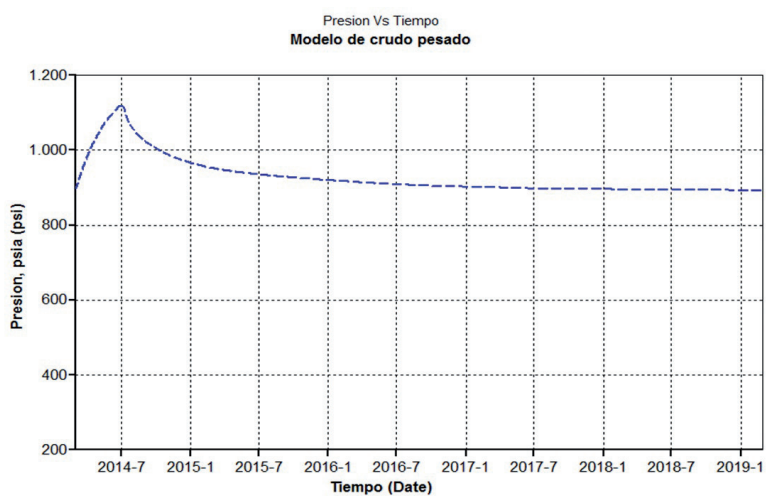

Figura 10. Comportamiento de la presión Vs tiempo, para el yacimiento de crudo pesado con movilidad

Fuente: Results Graph, CMG

Al comparar el comportamiento del proceso y expansión de la cámara de vapor se observa que aunque se tenga un yacimiento con las mismas propiedades petrofísicas si se desea aplicar la técnica de drenaje gravitacional asistida con vapor a un yacimiento con crudo pesado con movilidad es necesario monitorear, ajustar y/o cambiar algunos parámetros operacionales y de diseño para lograr resultados más favorables del proceso y poder así, alcanzar altos factores de recobro con SOR's bajos.

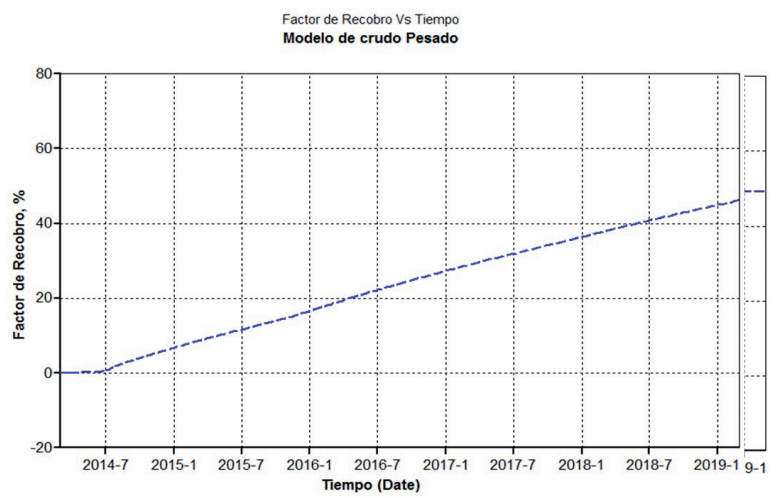

Figura 11. Factor de recobro Vs tiempo para el yacimiento de crudo pesado

Fuente: Results Graph, CMG

\section{CONCLUSIONES}

- La técnica de drenaje gravitacional asistida con vapor genera resultados exitosos cuando es implementada a crudos extrapesado, pues debido a la baja movilidad de este hidrocarburo a causa de su alta viscosidad, el vapor que se inyecte continuamente a la formación genera una cámara de vapor, la cual al expandirse tanto lateral como verticalmente permite contactar gran área del yacimiento, lo cual genera una reducción de la saturación remanente de petróleo, lo cual se ve representado en altos factores de recobro, manteniendo bajas relaciones de vapor aceite, lo cual corrobora la viabilidad técnica y económica del método en este tipo de yacimientos.

- Al implementar esta técnica en yacimientos de crudos pesados con movilidad, así este tenga excelentes propiedades petrofísicas, los resultados no son tan representativos como en los crudos extrapesados, esto a causa de que las fuerzas viscosas generan un efecto sobre la producción de los fluidos y la expansión del vapor en la formación, por lo cual es necesario evaluar su implementación en crudos móviles pues se requiere un ajuste en las variables operaciones y de diseño de la técnica y con ello, una redefinición de las propiedades de la formación bajo las cuales esta técnica pueda ser aplicada.

\section{AGRADECIMIENTOS}

Grupo de Recobro Mejorado GRM- UIS

\section{TABLA DE CONVERSIONES}

\begin{tabular}{ccc} 
& Sistema Ingles & Sistema Internacional \\
\hline Volumen & $1 \mathrm{Bbl}$ & $0,1589873 \mathrm{~m}^{3}$ \\
\hline Área & $1 \mathrm{Acre}$ & $4046,856 \mathrm{~m}^{2}$ \\
\hline Longitud & $1 \mathrm{ft}$ & $0.3048 \mathrm{~m}$ \\
\hline Temperatura & $1^{\circ} \mathrm{F}$ & $-17.22^{\circ} \mathrm{C}$ \\
\hline Presión & $1 \mathrm{Psi}$ & $6894,76 \mathrm{~Pa}$ \\
\hline Viscosidad & $1 \mathrm{cP}$ & $10^{-3} \mathrm{~Pa} . \mathrm{s}$ \\
\hline
\end{tabular}

\section{REFERENCIAS}

1. A.K SINGHAL, S.K. DAS. Screening of reservoirs for exploitation by application of steam assisted gravity drainage/vapex processes. SPE 37144, 1996.

2. BUTLER, R.M. Steam Assisted Gravity Drainage: Concept, Development, Performance and future. JCPT, 1994.

3. CHAKRABARTY C., RENARD G., FOSSEY J., GADELLE C. SAGD Process in the East Senlac Field: From Reservoir Characterization to Field Application. UNITAR Conference, Beijing, China, 1998.

4. CLARK Brian. Heavy Oil, Extra-Heavy Oil and Bitumen Unconventional Oil. NPC Global Oil and Gas Study. Julio 18-2007. 
5. DUSSEAULT Maurice. Diplomado en crudos pesados. Modulo V: "Gestión de Proyectos para el Desarrollo de Campos de Crudo Pesado y Nuevas Tecnologías". Bucaramanga 2008.

6. DUSSEAULT Maurice. Hydrocarbon from NonConventional Sourcer: Heavy and Extra Heavy Oil. Canada: Waterloo University. Cap 2.1.

7. ERIK MONTES \& HECTOR PACHECO; "Aplicación de nuevas tecnologías para la recuperación de crudo pesado en yacimientos profundos"; Tesis de grado; UIS 2006

8. GRILLS \& VANDAL. Case History: Horizontal well SAGD Technology is Successfully applied to produce Oil at LAK RANCH in Newcastle Wyoming. SPE 78964.

9. GUTIÉRREZ H., DE LA VARA R. Análisis y Diseño de Experimentos. $2^{\circ}$ Edición. México, 2008

10. HERNÁNDEZ SAMPIERI, R y otros. Metodología de la investigación. Cuarta edición. 2006.

11. HUMBERTO A. MENDOZA, JOSE J. FINOL \& ROGER M. Butler. "SAGD, Pilot Test in Venezuela"; SPE - 53687.
12. MERCADO C, ROSENDO M. "Recobro Mejorado de Crudos en Pozos Horizontales mediante el Método de Segregación Gravitacional Asistida por Vapor utilizando un solo pozo (SWSAGD). Tesis de grado. UIS. 2001.

13. SANDOVAL R., JIMENEZ R. "Nuevas Tecnologías para la Explotación de Yacimientos de Crudo Pesado". Informe de Investigación. Grupo de Investigación Recobro Mejorado (GRM). 2005.

14. SINGHAL, Y. ITO, M. KASRAJE. Screening and Desing criteria for Steam Assisted Gravity Drainage (SAGD) Projects. SPE 50401, 1998.

15. TERRATEK \&F PORTILLO; "Mechanical and Thermal Properties of Unconsolidated Sands and Its Applications to the Heavy Oil SAGD Project in the Tia Juana Field, Venezuela"; SPE - 54009.

16. TRIGOS E. Evaluación técnica de la factibilidad de implementar un proceso SAGD en yacimientos de crudo pesado. Universidad Industrial de Santander. 2010.

Recepción: 21 de enero de 2016

Aceptación: 20 de abril de 2016 